\title{
Bibliographie de la Société de Notre-Dame de Montréal (1639-1663) accompagnée de notes historiques et critiques (suite et fin)
}

\section{Marie-Claire Daveluy}

Volume 17, numéro 1, juin 1963

URI : https://id.erudit.org/iderudit/302271ar

DOI : https://doi.org/10.7202/302271ar

Aller au sommaire du numéro

Éditeur(s)

Institut d'histoire de l'Amérique française

ISSN

0035-2357 (imprimé)

1492-1383 (numérique)

Découvrir la revue

Citer ce document

Daveluy, M.-C. (1963). Bibliographie de la Société de Notre-Dame de Montréal (1639-1663) accompagnée de notes historiques et critiques (suite et fin). Revue d'histoire de l'Amérique française, 17(1), 141-152.

https://doi.org/10.7202/302271ar 


\title{
BIBLIOGRAPHIE*
}

\author{
Bibliographie de la Société de Notre-Dame de Montréal \\ (1639-1663) \\ accompagnée de notes historiques et critiques
}

\section{DEUXIÈME PARTIE}

\author{
Bio-bibliographie des Associés de Montréal \\ (suite)
}

Année 1648

45.-Ailleboust Des Musseaux (Charles d'), seigneur d'Argenteuil au Canada (1624-1700).

$$
\text { (suite) } \text { *** }^{*}
$$

Armes des d'Ailleboust, tel que consignées dans l'armorial de Rietstap: "De gueules au chevron d'or, accompagné de trois étoiles du même, deux en chef et une pointe $\gg^{47}$

\section{B. - ÉCRITS PERSONNELS}

Les Archives judiciaires de Québec et de Montréal contiennent un bon nombre de pièces diplomatiques signées par Charles d'Ailleboust des Musseaux. On doit aussi consulter les Papiers d'Ailleboust aux Archives de l'Hôtel-Dieu de Québec, relativement aux pièces concernant la succession de Louis d'Ailleboust de Coulonge.

* Voir notre Revue, V : 139-147, 296-307, 445-460, 603-616; VI: 146-150, 297-306, 458-463, 595-605; VII : 457-461, 586-592; VIII: 292-306, 449-455, 591-606; IX : 141-149, 306-309, 458-462, 594-602; X : 295-302; XI : 137-142, 298-304, 449-457, 608-614; XII : 144-147, 294-302, 443-453; XIII : 137-149, 298-305, 450-460, 594-602; XIV : 142-149, 302-311, 626-635; XV: 141-154, 466-472, 611-616; XVI: 294-307, 455-463, 608-612.

** Nous nous excusons d'avoir laissé tomber, au dernier moment, cette page ultime de la notice biographique de M. d'Ailleboust.

47 Nous retrouvons ces armes dans les armoiries épiscopales de S. Exc. Mgr Conrad Chaumont. Un écusson, placé en cœur, les reproduit et orne admirablement le blason de cet évêque distingué. 


\section{C. - NOTES BIBLIOGRAPHIQUES}

Dans son ouvrage sur la Famille d'Ailleboust (Montréal, 1917), Aegidius Fauteux a consacré une longue et excellente notice à Charles d'Ailleboust des Musseaux. Du reste, toute l'œuvre est une étude fouillée, fortement documentée sur les quatorze enfants de Charles d'Ailleboust et Catherine Le Gardeur de Repentigny et leur postérité.

Quant aux autres ouvrages à consulter, nous prions nos lecteurs de se reporter aux Notes bibliographiques concernant la vie et l'œuvre de Louis d'Ailleboust de Coulonge.

\section{Année 1661-1662}

46.-Conti (Armand de Bourbon, prince de), 1629-1666.

\section{A. - Notes BIOGRAPHiQUES}

Né à Paris le 11 octobre 1629 et fils cadet de Henri II, prince de Condé (1588-1646), et de Charlotte-Marguerite de Montmorency (1593-1650), c'est le chef de la branche de Conti. Son frère aîné, Louis II de Bourbon (surnommé le Grand), prince de Condé, premier prince du sang et duc d'Enghien, comptait 8 années de plus que son cadet, et sa sœur "la fougueuse », Anne-Geneviève de Bourbon-Condé, duchesse de Longueville, onze années de plus. Tous deux exercèrent plus tard une fâcheuse influence sur le prince de Conti. Sa famille, comme c'était alors la coutume, l'avait d'abord destiné à l'Eglise, « à cause de quelque défaut dans la taille et une certaine faiblesse de corps ». Son père s'empressa de le pourvoir de riches bénéfices ecclésiastiques. Il eut les abbayes de Saint-Denis, de Cluny, de Lérins et de Molesmes. Il fit de fortes études chez les Jésuites au Collège de Clermont; puis il apprit la théologie sous la direction du Père Etienne de Champ, jésuite. Jusqu'à la mort de son père, le prince demeura à l'Hôtel de Condé menant l'existence d'un ecclésiastique mondain. $M$. le prince, son père, peu de temps avant sa mort, avait demandé à Rome, pour son fils cadet, le chapeau de cardinal. Cependant une fois son père décédé, le prince se hâtait de quitter l'Eglise et d'entrer dans l'armée. Deux ans plus tard, il se jetait dans les intrigues de la Fronde étant sous l'entière domination de sa sœur, la duchesse de Longueville. Il combattit la reine et son ministre, le Cardinal Mazarin, que son frère, "le grand Condé 》, défendait alors. Puis, les deux frères, à la demande de leur mère, se réconcilièrent, 
et réunirent leurs forces contre Anne d'Autriche et son ministre. En 1650 les princes furent arrêtés et conduits à Vincennes. Le prince de Conti en sortit trois ans plus tard ayant accepté les propositions de Mazarin qui désirait lui faire épouser une de ses nièces. Effectivement, il s'unissait, en 1654, à Anne-Marie Martinozzi (1637 ?-1672), âgée de 16 ans, fille de Jérôme, comte romain, et de Laure-Marguerite Mazarini. Les époux se convertirent bientôt à la foi janséniste «mais avec cette nuance, pour le Prince, explique le Père Rapin, jésuite, (Mémoires, II: 367) qu'il y adhérait moins pour la doctrine qu'il n'approuva point étant théologien, que pour la morale qu'il estimait par le caractère de son esprit, excessif en toutes choses comme celui de la duchesse de Longueville, sa sœur ». Nous verrons toutefois le prince abjurer ses erreurs à la fin de sa brève existence. Le prince de Conti converti tout comme sa femme devint alors l'ami de saint Vincent de Paul, lui offrant plus d'une fois leurs services. A la mort du saint, le 27 septembre 1660 , il se fit un devoir d'assister à ses funérailles. Saint Vincent avait apprécié la piété sincère du prince. «Monseigneur le prince de Conti, écrivait-il dans une lettre datée du 13 août 1660 (voir la Correspondance du saint, éd. Pierre Coste, VIII: 368, lettre no 3198), sera un jour notre juge, au moins le mien. Il est admirable en sa fidélité à l'oraison; il en fait tous les jours deux heures, l'une le matin, l'autre le soir; et quelques grandes occupations qu'il ait et quelque monde qui l'environne, il n'y manque jamais [...] Plaise à Dieu de nous donner cet attrait pour nous unir à Notre-Seigneur [...].»

Si nous pouvons joindre le nom de ce grand seigneur, fils de Charlotte Marguerite de Montmorency, elle-même associée de Montréal, à ceux des nombreux Messieurs de Montréal, c'est grâce au Mémoire de $M$. d'Allet, de la Compagnie de SaintSulpice, le fidèle secrétaire de M. de Queylus, qu'il suivit du Canada en France à l'automne de 1661. M. d'Allet raconte «qu'aux assemblées de la Société de Notre-Dame de Montréal qui se tenaient depuis la mort de M. Olier (avril 1657), dans la chambre où celui-ci avait expiré, avoir vu, en qualité de président ou directeur, entrant ou sortant de charge, en 1661 et 1662, le pieux prince de Conti ...» (Voir le Mémoire de Messire Antoine d'Allet, p.s.s., dans les Oeuvres d'Arnault, tome $34 \mathrm{e}$ (Lausanne, 1775-1782). Cité par M. Faillon, Histoire de la colonie française, II : 271-276 et passim. Voir aussi notre note dans cette Bibliographie, première partie, no 45.

Nous devons rappeler ici l'intérêt profond que porta la princesse de Conti au Canada, intérêt entretenu par son mari 
tant qu'il vécut, puis plus tard, et jusqu'à la mort de cette princesse, par l'intendant Talon, «lequel, dit un historien canadien, ne s'en montrait pas peu fier ». Dollier de Casson, dans son Histoire du Montréal (éd. Flenley, p. 338), signale la générosité de "feue Madame la Princesse de Conty 48 [laquelle] a bonne part, écrit-il, avec quelques autres personnes de qualité à l'instruction de ces deux filles [deux jeunes Iroquoises élevées à l'européenne chez les Sœurs de la Congrégation de Notre-Dame, à Montréal], pour certaine somme de 12 ou 13 cents livres que leur charité avait envoyé l'automne dernier et qu'on eut soin d'employer selon leur pieuse intention ».

N'omettons pas non plus dans cette esquisse consacrée à Armand de Bourbon-Conti, l'élogieux portrait qu'en trace le Père Rapin, jésuite, dans ses travaux sur l'époque du jansénisme (voir surtout ses Mémoires, éd. Aubineau, II : 196 et passim) : «Ce prince, dit le Père, avait toutes les qualités d'un grand seigneur; il était brave, libéral, magnifique, méprisant les richesses qu'il n'amassoit que pour les répandre, prenant plaisir à donner par une grandeur d'âme qui n'a point presque eu d'égale en ce siècle, ne connoissant point de plus grand plaisir que celui de faire des heureux, ce qui attiroit à sa suite ce qu'il y avoit dans le royaume d'honnêtes gens qui se distinguoient par la bravoure ou par la science. Sa cour était remplie de gens d'esprit: les abbés de Cosnac, Roquette, [...], le Père Esprit, Voiture, Molière....» Les érudits bibliographes français, Messieurs Emile Bourgeois et Louis André, dans leur ouvrage: Les sources de l'Histoire de France, 3e partie, le XVIIe siècle (1610-1715) (Paris, Picard, 1923), «déclarent que l'un des beaux titres de gloire du prince de Conti est d'avoir protégé Molière $\gg^{49}$ Très instruit, le prince fut un écrivain apprécié à

48 Il faut se souvenir que M. Dollier écrivait son Histoire du Montréal de l'automne 1672 au printemps de 1673, peut-être même un peu après durant l'été. La princesse de Conti est morte, à Paris, le 4 février 1672 (voir Rapin, op. cit., II : 198, note 2).

49 S'il en est ainsi, Molière ne se serait pas montré fort reconnaissant envers son bienfaiteur. Car d'après l'abbé Mourret, dans son Histoire générale de l'Eglise, (VI: 173) : "Ce ne sont point les Jansénistes pas plus que les Jésuites que Molière voulut mettre en scène en 1668, dans son Tartuffe, ce sont les Confrères du Saint-Sacrement, c'est le prince de Conti... Molière avait peut-être aussi des rancunes à satisfaire contre ceux qui faisaient baisser les recettes de son théâtre, comme par exemple M. Olier, dans la paroisse de Saint-Sulpice, et le prince de Conti dans le gouvernement du Languedoc... En tout cas, [...] la comédie de Molière est une pure calomnie !» L'abbé Mourret a oublié, dans ces quelques lignes, avouons-le, que M. Olier et le prince de Conti, étant morts tous deux en 1668 , la vengeance de Molière s'exerçait dans le vide. 
son époque. Cet associé de la dernière heure à la Société de Notre-Dame de Montréal mourut à Pézenas, dans son gouvernement de Languedoc, le 21 février 1666. Il ne comptait que 47 ans.

Deux fils étaient nés de son union avec Anne-Marie Martinozzi : l'aîné, Louis-Armand de Bourbon, prince de Conti, «admirablement élevé par sa mère », donna, durant queiques années, de grandes espérances à la Cour, mais il mourut de la petite vérole en 1685, puis, François-Louis de Bourbon, né en 1664, qui fut roi de Pologne en 1697 et détrôné, peu d'années après, par un rival; il revint en France pour y mourir prématurément en 1707, à l'âge de 42 ans. Marié, il laissait un fils qui continua la lignée des Bourbon-Conti.

Armes des Bourbon-Conti: Ecartelé: aux 1 et 4 de France, au bâton de gueules péri en bande, qui est de Bourbon; aux 2 et 3 de France, à la bordure de gueules chargée de huit besants d'argent, qui est d'Alençon.

\section{B. - ÉCRITS PERSONNELS}

Nous laisserons de côté les nombreuses pièces d'archives, narratives et diplomatiques. Nous ne mentionnerons que les ouvrages imprimés peu après sa mort.

262.- Traité de la comédie et des spectacles selon la tradition de l'Eglise. Paris, 1666.

263.-Devoirs des grands, avec son Testament. Paris, 1666, in-8.

264.-Devoirs des gouverneurs de province. Paris, 1667. 3 vol. in-12.

$$
\text { C. - Notes BIBLIOGRAPHiques }
$$

N.B. Ici encore, nous nous bornerons à citer les sources biographiques et littéraires fondamentales.

265.-Voisin, Joseph de, Abrégé de la vie du prince de Conti, dans son ouvrage Défense du traité de Monseigneur le prince de Conti contre la Comédie. Paris, 1672. in-4.

N.B. Les bibliographes Emile Bourgeois et Louis André, dans les Sources de l'Histoire de France, 3e partie, le XVIIe siècle (III: Biographies: 172), nous présentent cette note sur l'auteur de l'Abrégé de la vie du prince de Conti: «Voisin était l'aumônier du prince; il a résumé les peu nombreuses actions qui ont marqué la vie de Conti. » 
266.-Choiseul, Monseigneur Gilbert de, évêque de Comminges, 1613(?)-1689, Oraison funèbre [du prince de Conti]. Paris, 1666, in-4, 40 pages.

Rappels: Voir dans notre Bibliographie, au no 179, la description de l'ouvrage du Père Rapin, s.j., Mémoires ..., éd. Léon Aubineau, 1865. 3 volumes. Voir aussi du même auteur son Histoire $d u$ jansénisme, éditée par l'abbé Domenech (Paris, 1861), in-8.

\section{REMARQUE FINALE CONCERNANT LES ASSOCIÉS DE MONTREAL}

Avant de clore notre liste des Associés de Montréal, nous considérons comme un devoir d'y ajouter quatre autres noms, dont nous espérons pouvoir justifier l'inclusion. Du reste, trois parmi ces noms ont été inscrits dans la liste de l'érudit Jacques Viger. ${ }^{50}$ Et le non moins savant abbé Hospice-Anthelme Verreau, tout en ne les mentionnant point dans sa propre liste, les énumère dans une note sans y apporter d'objection. Il écrit même ceci: « Cette liste [celle de Jacques Viger] diffère très peu de celle qui précède [la sienne]; et, si l'on considère que nous avons puisé à d'autres sources que M. Viger, on ne pourra s'empêcher d'admettre que l'accord entre les deux listes est une présomption très forte en faveur de l'exactitude de l'une et de l'autre. \51

Voici les trois associés probables auxquels nous nous permettons d'ajouter un quatrième:

1. Jean de Lauson, quatrième gouverneur de la NouvelleFrance;

2. Jacques Girard, seigneur de La Chaussée et de La Callières ;

3. Marguerite Bourgeoys (la bienheureuse) ;

4. Guillaume de Lamoignon, premier président du Parlement de Paris, magistrat célèbre.

ARGUMENTS ET DÉDUCTIONS HISTORIQUES pouvant rendre plausible la présence des quatre associés que nous allons maintenant placer dans leur ordre chronologique d'entrée.

50 Voir les Mélanges religieux (1842) : 389, note.

51 Voir l'introduction que M. Verreau écrivit en éditant les Véritables Motifs des Messieurs et Dames de la Société de Notre-Dame de Montréal [...] Montréal, 1880, XLVII (47) pages [...] (Mémoires de la Société historique de Montréal, 9e livraison). 
1.-Année 1640: Lauson (Jean de), (1584-1666).

Conseiller au Parlement de Paris (1613), maître des requêtes (1622), intendant dans le Dauphiné (1640-1646), premier président du Conseil de direction de la Compagnie des CentAssociés (1627), quatrième gouverneur de la Nouvelle-France (1651-1653) ; obtient des provisions pour un second terme (16541657), mais retourne en France en 1656, abandonnant l'administration du pays à son fils, Charles de Lauson-Charny. Fut l'ami des Messieurs d'Ailleboust, l'oncle et le neveu. «S'était taillé un royaume dans la Nouvelle-France », déclare JosephEdmond Roy dans son ouvrage, La Seigneurie de Lauzon (Lévis, $1897-1904,5$ vol.). D'après le Père Ragueneau, jésuite, son ami, Jean de Lauson mourut à Paris le 16 février 1666. Il comptait 70 ans quand il quitta le Canada.

N.B. Rappelons d'abord que M. de La Dauversière, pressé d'acquérir l'île de Montréal pour y fonder une colonie, accomplissait, dans l'été de 1640 un second voyage dans le Dauphiné auprès $d u$ propriétaire de l'île, l'intendant Jean de Lauson. ${ }^{52}$ Fort heureusement il était accompagné cette fois par le Père Charles Lalemant, jésuite, un ami de l'intendant. L'intervention du Père Lalemant fut victorieuse des refus obstinés jusqu'ici de M. de Lauson. Alors, comment ne pas admettre que M. de La Dauversière, au comble de ses vœux, ait songé à inviter $M$. de Lauson à faire partie de la Société de Notre-Dame de Montréal ? Le geste vraiment s'imposait tant au point de vue diplomatique qu'à celui de la plus élémentaire courtoisie.

Dans ses Ecrits autographes, Mère Bourgeoys relate le fait et donne plusieurs détails intéressants. (Voir sa Vie éditée par M. de Montgolfier, p.s.s., en 1818: 18-22). Sans doute, comme dans tous les Mémoires, il y a, dans cet ouvrage, des erreurs de dates et des imprécisions, mais l'ensemble des faits demeure véridique.

2.-Année 1640: GIRARD (Jacques), seigneur de La Chaussée et de La Callières.

N.B. Ce fut l'ami de Jean de Lauson, un de ses prête-noms, dans l'acquisition des immenses concessions que celui-ci posséda dans la Nouvelle-France, notamment la seigneurie de La Citière

52 Nous adoptons la forme du nom Lauson (non Lauzon) conformément à cette orthographe du nom que nous trouvons dans le deuxième Mémoire de la Société historique de Montréal (Montréal, 1859), intitulé: De la famille des Lauson, par Sir Louis-Hippolyte LaFontaine. 
qui contenait l'île de Montréal. En sa qualité de membre et de directeur de la Compagnie des Cent-Ássociés, M. de Lauson ne pouvait acquérir lui-même aucune propriété au Canada. En outre, une clause du Contrat que signa son prête-nom, stipulait que l'acquéreur devait s'occuper de coloniser ses concessions de La Citière, sous peine de voir annuler son contrat. Or, lors de la Cession de l'âle de Montréal, par acte daté du 7 août 1640, M. de Lauson n'avait encore commencé aucun des travaux propres à la colonisation de l'île. La Compagnie des Cent-Associés, devant toutes ces illégalités, annula l'acte de cession du 7 août 1640 et vendit elle-même, par un nouveau contrat signé en décembre 1650, l'île de Montréal aux procureurs de la Société de NotreDame, MM. de La Dauversière et Fancamp.

Ces incidents fâcheux mirent forcément le prête-nom de M. de Lauson, M. Girard de La Chaussée, en relations avec MM. de La Dauversière et Fancamp. Ceux-ci ne pouvaient qu'inviter M. de La Chaussée à se joindre aux membres de la Société de Montréal, tout comme son ami, M. de Lauson. Les Ecrits autographes de Mère Bourgeoys laissent clairement entendre que les choses se passèrent ainsi autour des contrats d'août et de décembre 1640.

\section{3.-Année 1653: LamoignoN (Guillaume de), (1617-1677).}

Premier président au Parlement de Paris, en 1658, magistrat éclairé et recherché, marquis de Basville, comte de LaunaiCourson, et baron de Saint-Yon. Fils de Chrestien de Lamoignon et de Marie des Landes, que l'on surnommait à Paris la mère des pauvres. ${ }^{53}$ Parent de Claude de Bullion dont la mère était née Charlotte de Lamoignon, Guillaume fut très estimé de Madame Angélique Faure de Bullion, la bienfaitrice inconnue du Montréal. Ce fut à ce savant gentilhomme que Madame de Bullion voulut remettre, en 1652, une somme substantielle pour aider au recrutement des colons-soldats qui allait sauver bientôt Ville-Marie, et par ce poste exposé, toute la Nouvelle-France. Le texte de Dollier de Casson (Histoire du Montréal, éd. Flenley, 1928, 187-188) au sujet de cette intervention, du président de Lamoignon auprès de la Société de Montréal, nous permet de croire qu'il y appartenait. Si Madame de Bullion ne s'était pas nommée en remettant son don à M. de Lamoignon, si même elle s'efforça de lui faire croire qu'elle n'y était pour rien, pourquoi

53 Lorsque cette femme admirable mourut, tous les pauvres du quartier s'insurgèrent et empêchèrent son inhumation dans l'église des Cordeliers de Paris où se trouvait le tombeau des familles Bullion et Lamoignon. On dut l'enterrer dans l'église de sa paroisse. 
l'aurait-elle choisi au lieu de M. de Maisonneuve, par exemple, qui lui avait appris la situation désespérée du Montréal; oui, pourquoi l'avoir chargé d'une mission délicate auprès d'une société parfaitement inconnue du président. Elle ne pouvait non plus lui avoir parlé directement de M. de Maisonneuve, chef du recrutement des colons-soldats, c'eût été par trop se compromettre elle-même. En outre, la plupart des associés de Montréal comptait le président Lamoignon comme ami, tel Barillon de Morangis, Mêmes d'Irval, Bertrand Drouart, Chomedey de Maisonneuve, et sans doute aussi, par tous ceux-ci connut-il fort bien MM. de La Dauversière, Olier et Fancamp.

\section{4.-Année 1658: BourgeoYs (La Bienheureuse Marguerite), (1620-1700).}

La fondatrice de la Congrégation de Notre-Dame a été, nous le savons, l'une des grandes héroïnes du Montréal à l'époque héroïque. Amie de Jeanne Mance, conseillère appréciée par M. de Maisonneuve, tout Ville-Marie apprit peu à peu à la vénérer comme une sainte et une merveilleuse éducatrice en France. En 1658 elle s'offrait à accompagner Jeanne Mance, malade, invalide du bras droit depuis sa chute sur la glace en 1657. Marguerite lui faisait aussi part honnêtement que ce voyage lui serait fort utile pour recruter des compagnes pour la fondation de sa future communauté. Jeanne Mance fut ravie de ses propositions qui non seulement la favoriseraient, mais contribueraient également à l'établissement d'une solide institution pour l'enseignement des enfants français et sauvages. Marguerite Bourgeoys accomplirait ainsi l'un des vœux les plus chers de la Société de Notre-Dame de Montréal: instruire et civiliser les Sauvages.

Il est certain que Marguerite Bourgeoys, en France, ne quitta point Jeanne Mance les premières semaines de son arrivée. Elle l'accompagna d'abord à La Flèche, chez les Hospitalières de Saint-Joseph. Elle fit connaissance avec Jérôme Le Royer de La Dauversière qui dut lui témoigner toute sa joie de ce qu'elle songeait de plus en plus à sa mission d'éducatrice auprès des colons de Ville-Marie. Puis une fois à Paris, Marguerite continua quelque temps encore, à l'endroit de Jeanne Mance, son rôle de garde-malade. Elle assista certainement aux assemblées des Associés de Montréal, tous désireux d'entendre Jeanne Mance sur les progrès du Montréal, tout en déplorant son lamentable accident et en s'efforçant d'y remédier de quelque façon. L'occasion dut sembler providentielle à Jeanne de présenter sa fidèle compagne et amie, Marguerite Bourgeoys, comme une 
ouvrière des plus zélées en tout ce qui touchait le bien-être des Sauvages dont elle s'était faite l'institutrice dévouée. Et ces Messieurs de s'en réjouir, certes, et de féliciter l'intelligente et pieuse Marguerite de ses travaux. Elle était vraiment une véritable associée de Montréal pour ces bienfaisants gentilshommes qui la voyaient mener à bien un des grands projets de la Société de Notre-Dame concernant les Sauvages.

Puis, souvenons-nous qu'à partir de ce voyage en France, Marguerite Bourgeoys entretint de constantes relations avec plusieurs de ces Messieurs, notamment le baron de Fancamp et les Messieurs Le Prêtre. Si les Ecrits autographes de la bienheureuse ne font aucune allusion à sa qualité d'associée de Montréal, c'est que sa rare humilité lui imposait là-dessus le silence.

MM. Viger et Verreau, dirons-nous encore, approuvèrent implicitement les réflexions de $M$. de Montgolfier, supérieur de Saint-Sulpice, au sujet du nom de Marguerite Bourgeoys, à ajouter aux membres les plus agissants de la Société de NotreDame. M. de Montgolfier écrit dans la Vie de la Vénérable Sour Marguerite Bourgeois, dite du Saint-Sacrement ${ }^{54}$ (A VilleMarie: Chez Wm. Gray, rue St-Paul, 1818, 20) : «Et la vénérable sœur Marguerite Bourgeois, dont on donne ici la vie, qui, sans être formellement du nombre des associés [ - tout comme Mme de Bullion, par exemple - ] a eu plus de part que qui que ce soit à l'exécution de la bonne œuvre. Nous la verrons bientôt paraître, cachant l'éclat de ses vertus sous une humilité profonde. 》

On ne saurait s'exprimer de façon plus sûre, plus concluante sur le sujet qui nous occupe en ce moment.

\section{CONCLUSION}

La Société de Notre-Dame de Montréal veilla, durant un quart de siècle, sur la cité de Ville-Marie. L'histoire des origines religieuses de notre ville constitue un chapitre substantiel de l'histoire de l'Eglise au Canada. Mais dans notre étude, avonsnous suffisamment insisté sur l'extrême péril, les obstacles sans nombre qui entravèrent les travaux, sur la précarité sans cesse accrue de ce poste exposé de la Nouvelle-France. Surtout avonsnous loué, comme il convenait, la foi profonde, malgré l'insuccès,

54 Dite du Saint-Sacrement !... Le Saint-Sacrement ! La grande dévotion, avec celle de la Sainte Vierge, des Messieurs de Montréal, la plupart membres de la Compagnie du Saint-Sacrement. L'intelligente Sœur Marguerite songeait certes à ces beaux souvenirs mystiques en choisissant son nom particulier de religieuse dans la Congrégation de Notre-Dame. 
de ceux qui tinrent le coup pendant si longtemps. Inclinons-nous du moins une dernière fois devant l'état d'esprit et les élans spirituels grandissants des Associés de Montréal.

Car tous les courants de mysticité, tous les mouvements de charité dignes des âges apostoliques qui marquèrent le sentiment religieux du grand siècle, baignent de leur ferveur l'atmosphère de la Société. Tout d'abord, il faut rattacher celle-ci à la Compagnie du Saint-Sacrement. Son fondateur, le duc de Ventadour, ex vice-roi de la Nouvelle-France, et quelques autres membres, Jérôme Le Royer de La Dauversière, Jean-Jacques Olier, Gaston de Renty, Antoine Barillon de Morangis, Christophe du Plessis-Montbard et Jean de Garibal, se préoccupèrent de Ville-Marie avec le même zèle, la même constance et les mêmes moyens d'exécution que s'il se fût agi d'une filiale de la célèbre Compagnie. Ils l'entourèrent d'une armature de silence et d'anonymat, d'ampleur dans les dons, et du plus minutieux examen des «véritables motifs » de l'action à exercer.

Le branle initial fut donné par Jérôme Le Royer de La Dauversière, l'ascète prodigieux, l'inspiré, le saint homme de La Flèche. Et cette constatation nous permet de croire que la fondation de Montréal fut la réalisation de vues providentielles. A bien y songer, que ne dut pas Montréal à ce Fléchois infatigable. Le véritable fondateur de notre ville vécut et mourut à la peine, œuvrant héroïquement pour cette Ville-Marie dont l'existence traversée de tragiques événements semblait vouée à la mort. Hélas, la dette que nous avons contractée envers un tel apôtre demeure encore impayée par nous.

Il faut également reconnaître que c'est à M. de La Dauversière, conseillé en cette occasion par les Jésuites, maîtres vénérés de sa jeunesse, que nous devons le chef de Ville-Marie, ce Paul de Chomedey de Maisonneuve dont la droiture, le désintéressement et la foi furent ceux d'un saint plus encore que d'un héros. Et c'est encore M. de La Dauversière qui précisa, à la Rochelle, à l'heure de l'embarquement de la première recrue montréalaise, la mission de Jeanne Mance au Canada. Cette femme de tête et d'initiative, au cœur d'une inépuisable charité, mérita, par son étroite collaboration aux vues de $M$. de Maisonneuve, le titre de co-fondatrice de Montréal. Puis vinrent les fils de M. Olier, la bienfaisante Compagnie de Saint-Sulpice. Elle enveloppa de soins et combla de dons fastueux la cité de Ville-Marie. On se rappellera que Saint-Sulpice est né à l'époque même où naissait notre ville. Que serait devenu Montréal, après la mort de M. de La Dauversière, si Messieurs de Bretonvilliers, successeur du saint fondateur, Souart, premier curé de Ville- 
Marie, et de Queylus qui versa son or sans compter, n'eussent tendu des mains généreuses vers la ville de Notre-Dame.

Enfin, dans d'autres domaines, rappelons quelques faits où figurent de nouveau les Associés de Montréal. Ainsi, ne sait-on pas qu'à cette époque, le jansénisme s'infiltrait avec sa doctrine subtile dans tous les milieux dévôts. La Société de Notre-Dame n'échappa point à ce danger. Mais ce furent des cas d'exception que ceux où s'enlisèrent les Liancourt, le prince de Conti et quelques autres membres. Les ombres du jansénisme ne purent vaincre, dans les âmes éclairées des Messieurs et Dames de Montréal, la limpidité d'une foi aussi humble que réfléchie.

Evoquons maintenant un coin de tableau où apparaît le XVIIe siècle bien-disant et créateur de chefs-d'œuvre littéraires. De beaux génies chrétiens, des lettrés au regard lucide, des ironistes supérieurs expriment leurs vues pénétrantes sur la société de leur temps. On vit même des salons où se réunissaient maints penseurs, orateurs, poètes, hommes d'esprit et de goût, exercer une influence remarquable sur leurs contemporains. Et voici l'hôtel de Rambouillet et son souci de «la langue la plus délectable qui soit au monde $\gg .$. . Voici peu après la création de l'Académie française, ce cénacle de beaux esprits protégé par Richelieu. Ces milieux comptèrent dans leurs réunions des associés de Montréal. Parfois, ceux-ci sortaient à peine d'une assemblée de charité, où avait résonné la voix d'un saint Vincent de Paul, ou encore d'une cérémonie mi-religieuse, mi-missionnaire, de la Société de Notre-Dame, où l'on avait discuté de la conversion des Sauvages de la Nouvelle-France.

Comment ne pas croire, en se remémorant ces beaux faits, à la croissance continue, en force, en importance, en une certaine grâce pittoresque, en une vie spirituelle toujours montante, de cette cité de Ville-Marie trois fois centenaire ? Comment ne pas espérer en une future et longue survie, car Notre-Dame veille toujours sur la ville dont de grandes âmes décidèrent, en un jour de ferveur mariale, qu'elle en serait la souveraine toutepuissante et incontestée.

\section{MARIE-ClaIRE DAVELUY de l'Académie canadienne-française}

N.B. Comme nous nous proposons d'éditer, très prochainement, notre étude maintenant terminée, nous n'ajouterons ici aucun tableau explicatif, aucune liste d'ouvrages publiés depuis juin 1951. Nos lecteurs voudront bien se référer à l'ouvrage que nous publierons.

M.-C. D. 